\title{
A Multiview Facial Analysis Technique for Identity Authentication
}

\author{
Biometric systems, which use people's physiological characteristics for \\ identification or authentication, have become increasingly popular for \\ countering fraud. In this article, we describe a face recognition system \\ that uses a new distance measure for authentication. It performs \\ matching on a fusion of multiple views of each person.
}

\section{Yongsheng Gao Griffith University}

S.C. Hui Nanyang Technological University
A.C.M. Fong
Massey University

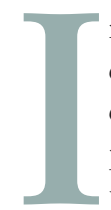

n Mark Weiser's seminal article on ubiquitous computing, he described a world in which computers work harmoniously and transparently with human users, serving human users in different aspects of their lives. ${ }^{1}$ Although some of his ideas seemed far-fetched at the time, many of the enabling technologies necessary for realizing Weiser's vision are now becoming available. For example, technological advancements in hardware mean that physical computing machines (such as personal computers) will gradually become invisible to human users because both mobile clients and infrastructure systems can work seamlessly and unobtrusively. ${ }^{2}$ You can already disguise mobile client devices by embedding them into personal belongings that people carry or wearhandbags, shoes, cellular phones, and so on. Similarly, software technology developments ensure the ready integration and interoperation of diverse systems. $^{3}$

However, many questions remain on how to effectively integrate these components-the building blocks of pervasive computing. ${ }^{4}$ In this article, we focus on using biometric-based authentication techniques to address privacy and trust issues.

\section{Biometrics for ubiquitous computing environments}

Traditional person recognition methods do not meet today's needs. Large sums of money have changed hands based on a signature, identity cards, or passwords-all unreliable or forgeable sources. Additionally, you can lose (through theft or absentmindedness) smart cards and similar devices (although you can reduce the risk by embedding them into things or eventually the human body ${ }^{5}$ ). So, the need increases for fast, accurate, and userfriendly automatic person recognition systemsideally, ones that don't need human attention. Such systems could address purposes such as physical access control (border control and access to secure areas), traditional business transactions, and even the management of surrogate computing resources, online transactions, and criminal investigations.

In recent years, biometrics has gained importance as a research area. ${ }^{6,7}$ Biometrics relates to a person's physiological features (fingerprints, face) and behaviors (speech), which you can use to distinguish one person from another. In fact, law enforcement agencies have used fingerprinting technology in forensics for many years. A biometric system should be nonintrusive to users. For example, most users would deem face or speech recognition more acceptable than an iris scan. 
Figure 1. Overview of our experimental system: (a) enrollment; (b) authentication (fused multiview analysis).

In our research, we focus on face recognition (see the related sidebar)-one of biometrics' least intrusive forms - and aim to promote the goal of invisibility mentioned earlier. You can classify face recognition as authentication and identification. In authentication, we try to answer the question "is the person claming to be person $X$ really person $X$ ?" This gives a binary decision. In identification, we want to find the person most likely to be $X$ from among a pool of candidates in a database.

Different performance metrics exist for authentication and identification. ${ }^{8}$ For identification, metrics focus on the likelihood that the correct answer appears in the list of top (say 10) matches; for verification, the primary performance measure is the tradeoff between false acceptance and false rejection rates, which leads to the consideration of an equal error rate line. Typically, we concentrate on plotting FRR against FAR to compare different systems' or system parameters' performance. This results in receiver operating characteristic curves. So, the line given by FRR = FAR in a ROC plot is the EER.

In our research, we focus on authentication. You can use it to manage access control to surrogate computing resources during cyber-foraging activities. Most research concentrates on frontal facial analysis. ${ }^{9,10}$ To improve system robustness, others have developed multimodal approaches that fuse together different biometrics. ${ }^{11}$ However, we believe optimizing a single-modal system minimizes deployment costs and could benefit any multimodal system.

You can improve a face recognition system's robustness by taking multiple views of a person's face at different angles using essentially the same equipment as frontalonly analysis. We developed a multiview face recognizer for authentication that meets these design objectives:

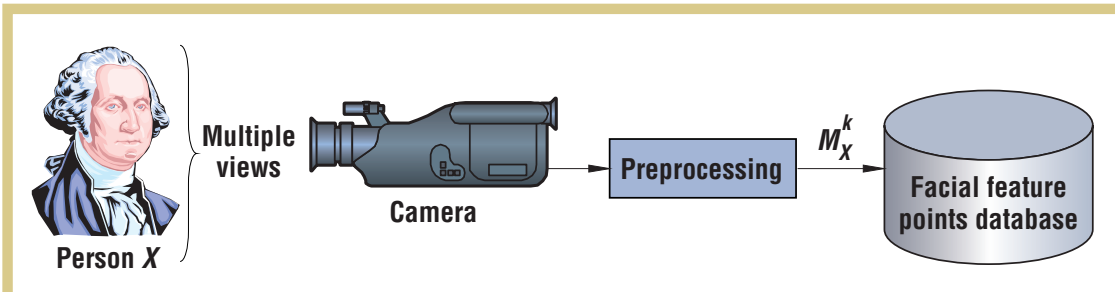

(a)

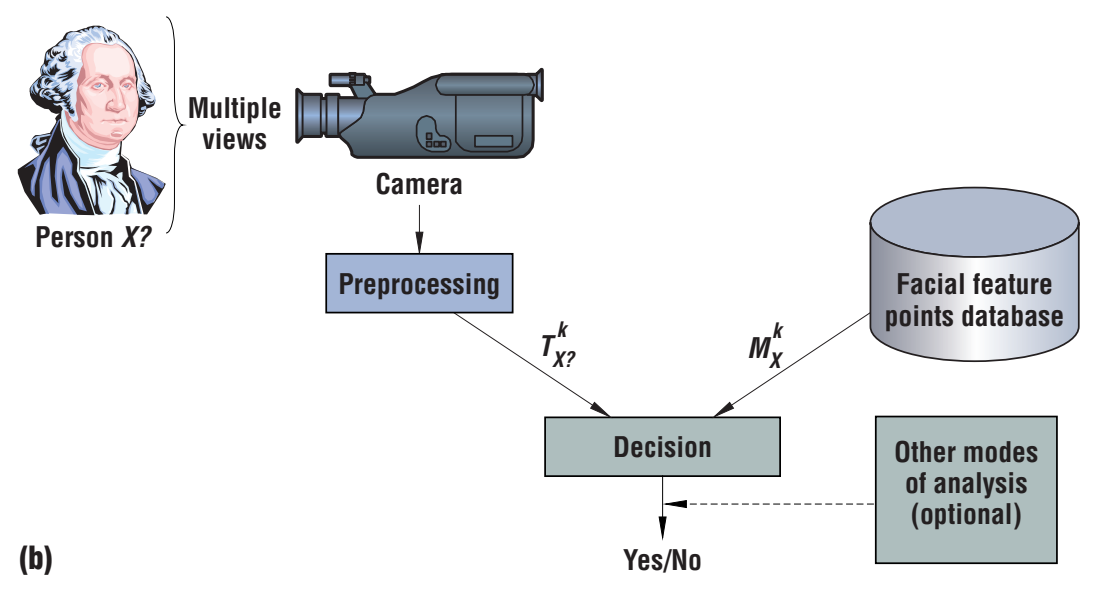

- Robust against variations. Operates under different conditions and applies to a high percentage of the population.

- Cost effective. Incurs minimal deployment costs.

- Accurate and reliable. Has excellent performance distinguishing between people and excellent EER performance.

- User-friendly. Is nonintrusive and fast.

As Figure 1 shows, we target our solution toward the authentication of users who are willing to have an enrollment process capture images of their faces. These captured images form a knowledge base used for subsequent authentication by comparing person $X$ 's stored image with the image of a person claiming to be $X$.

\section{Significance-based multiview Hausdorff distance}

Suppose a set of feature vectors (nodes) $N_{\mathrm{A}}$, or model points, represents an image $A$ and another set $N_{\mathrm{B}}$, or test points, represents image $B$. (See the "Face recognition" sidebar for more information.) Traditional methods that assign the same weight to all points in $N_{\mathrm{A}}$ and $N_{\mathrm{B}}$ clearly lead to suboptimal results because different feature points contribute differently toward an overall face description. In this article, we apply a new variant of Hausdorff distance called the significance-based multiview Hausdorff distance (see the "Face Recognition" sidebar).

SMVHD differs from other HD variants in two ways. First, we apply the notion of significance associated with each point. Second, we fuse together multiple views of the same nonrigid object (the face) taken from different viewpoints. (Using multiview images for facial analysis is not entirely new. The TrueFace system (see www.biometricgroup.com/a_bio1/vendor/ miros.html) uses two images. However, its reliance on neural network technology might account for a relatively long computational time-a claimed recognition time of 2 s.) More specifically, suppose

$$
\begin{aligned}
& M^{1}=\left\{m_{1}^{1}, m_{2}^{1}, \cdots, m_{p_{1}}^{1}\right\}, \\
& M^{2}=\left\{m_{1}^{2}, m_{2}^{2}, \cdots, m_{p_{2}}^{2}\right\}, \ldots,
\end{aligned}
$$




\section{Face Recoginition}

$\mathrm{E}$ igenface, elastic graph matching, and backpropagation neural nets are traditionally considered the three major approaches developed for face recognition. ${ }^{1,2}$ Because a comprehensive survey judged EGM the best of the three techniques, ${ }^{2}$ we won't discuss the other two techniques here. EGM entails the analysis of a face represented by a set of feature descriptors located at the nodes of a sparse grid (or lattice). 3,4 Due to relatively simple processing, the nodes are typically distributed evenly over a rectangular grid. However, you can also situate the nodes at important feature points (for example, eyes) called fiducial points.

Figure A illustrates the basic idea of EGM (using a rectangular grid). ${ }^{2}$ The grid's nodes form a set of feature vectors that mathematically describe the face's characteristics. Suppose we define a 2D image lattice $S$ and within it a smaller, coarser lattice $S_{1}$. The face template, which characterizes the face's features, is a vector field $\mathbf{c}=$ $\left\{c_{i}, i \in S_{1}\right\}$, where $c_{i}$ is a feature vector at node $i$. During recognition, we require another vector field called the face vector field $\mathbf{x}=\left\{\mathbf{x}_{j}, j \in\right.$ $S\}$. So, $\mathbf{x}$ is defined over the original finer lattice $S$. You obtain the distance $d(\mathbf{c}, \mathbf{x})$ by finding a best match $M$ between $\mathbf{c}$ and $\mathbf{x}$, where $M$ is a mapping between $S$ and $S_{1}$. EGM aims to minimize an energy (or cost) function $E(M)$.

Comparing two faces (between person $X$ 's stored image and a newly captured image of the person claiming to be person $X$ ) entails minimizing $E(M)$ in two steps. In step 1, as in conventional template matching without any defor-

Figure A. Illustration of elastic graph matching: (a) 2D image lattice $S$ and within it a smaller, coarser lattice $S 1$; (b) placing $\mathrm{S} 1$ on the face image. mation, moving $\mathrm{c}$ around $\mathrm{x}$ performs rigid matching. Once we find the position with the minimum distance, we obtain an initial match $M_{0}$ with energy $E\left(M_{0}\right)$. In step 2 , stretching $S_{1}$ performs deformable matching, to allow $\mathrm{c}$ to deform to further minimize the energy function. This flexibility provides robustness against changes in illumination and variations in nonrigid objects such as faces.

More recently, researchers have proposed other face recognition techniques such as linear discriminate analysis and support vector machines. ${ }^{5}$ In our research, we apply a technique called the dynamic two-strip algorithm (Dyn2S), ${ }^{6}$ which relies on detecting dominant points on the facial edges. This approach somewhat resembles EGM in that it extracts dominant points from a person's face to form a mathematical description of the face image instead of using a grid in a particular form.

Because we need to quantitatively analyze a match between two images, we must consider a suitable distance measure. For example, suppose a set of feature vectors (nodes) $N_{A}$, model points, represent an image $A$ and another set $N_{B}$, test points, represents image $B$. Then, matching images $A$ and $B$ amounts to determining whether the dis-

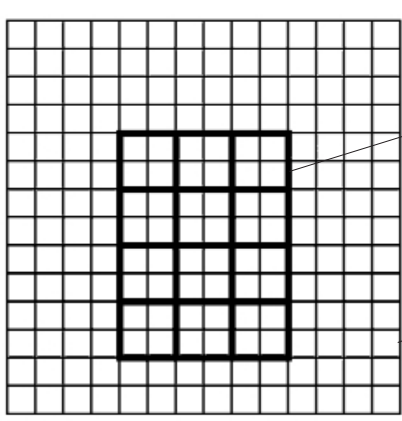

(a)

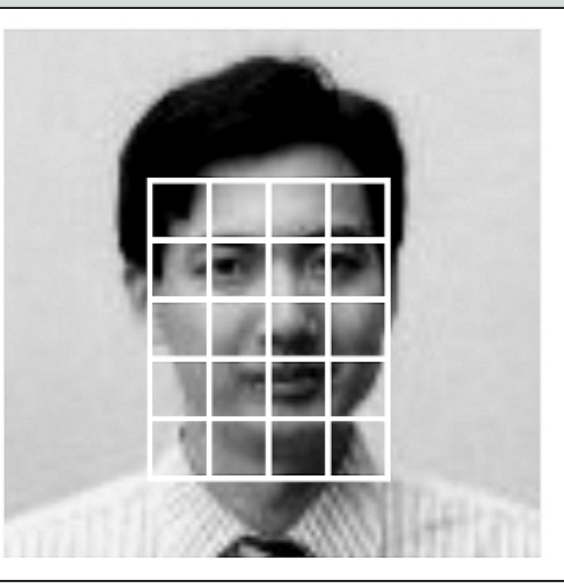

$M^{n}=\left\{m_{1}^{n}, m_{2}^{n}, \cdots, m_{p_{n}}^{n}\right\}$

are $n$ point sets representing the features in object M's $n$ model views and

$$
\begin{aligned}
& T^{1}=\left\{t_{1}^{1}, t_{2}^{1}, \cdots, t_{q_{1}}^{1}\right\}, \\
& T^{2}=\left\{t_{1}^{2}, t_{2}^{2}, \cdots, t_{q_{2}}^{2}\right\}, \cdots, \\
& T^{n}=\left\{t_{1}^{n}, t_{2}^{n}, \cdots, t_{q_{n}}^{n}\right\}
\end{aligned}
$$

are the corresponding $n$ point sets repre- senting the features in test object T's $n$ views from the same viewpoints as in the model $M$. In this formulation, $p_{1}, p_{2}, \ldots$, $p_{n}$ and $q_{1}, q_{2}, \ldots, q_{n}$ are the point numbers, which are used for indexing, in the model and test views. So, we must let only the $i$ th point in the $k$ th view point $t_{i}^{k} \in T^{k}$ match with the points in $M^{k}$, the $k$ th view of $M$ (see Figure 2).

We define the SMVHD between $M$ and $T$ as

$$
\begin{aligned}
H_{S M V H D}(M, T)= & \max \left(h_{S M V H D}(M, T),\right. \\
& \left.h_{S M V H D}(T, M)\right) .
\end{aligned}
$$

In turn, we define the directed SMVHD from $M$ to $T$ and from $T$ to $M$ as

$$
\begin{aligned}
& h_{S M V H D}(M, T)=\frac{1}{\sum_{k=1}^{n} \sum_{m_{i}^{k} \in M^{k}} S i g_{m_{i}^{k} t_{j}^{k}}} \\
& \times \sum_{k=1}^{n} \sum_{m_{i}^{k} \in M^{k}}\left(S i g_{m_{i}^{k} t_{j}^{k}} \bullet \min _{t_{j}^{k} \in T^{k}}\left\|m_{i}^{k}-t_{j}^{k}\right\|\right)
\end{aligned}
$$


tance between the respective nodes in $N_{A}$ and $N_{B}$ are sufficiently close. An authentication system makes a binary decision based on this proximity. So, an effective distance measure is critically important in determining a face recognizer's performance for authentication.

Hausdorff distance is a popular distance measure well suited to establishing similarity between two sets of points. ${ }^{7}$ Unlike most other methods that rely on a rigid point-to-point relationship between model and test points, you can determine HD without an explicit relationship. This makes HD particularly useful for comparing nonrigid objects. In fact, some commercial products-for example, BiolD ${ }^{8}$ — use HD for face location and eye location.

Mathematically, if we have two finite sets of feature points $M=$ $\left\{m_{1}, m_{2}, \ldots, m_{k}\right\}$ (representing a model) and $T=\left\{t_{1}, t_{2}, \ldots, t_{n}\right\}$ (representing a test image), we can define HD as

$$
H(M, T)=\max (h(M, T), h(T, M)),
$$

where

$$
h(M, T)=\max _{m_{i} \in M} \min _{t_{j} \in T}\left\|m_{i}-t_{j}\right\|
$$

and $\left\|m_{i}-t_{j}\right\|$ denotes the Euclidean norm on points of $M$ and $T$. The function $h(M, T)$ is the directed $\mathrm{HD}$ from $M$ to $T$. It identifies the point $m_{i} \in M$ that is the farthest from any point of $T$ and measures the distance from $m_{i}$ to its nearest neighbor in $T$. The Hausdorff distance $H(M, T)$ is the maximum of $h(M, T)$ and $h(T, M)$. So, it measures the degree of mismatch between two sets by measuring the distance of point $M$ that is farthest from any point of $T$ and vice versa.

Unfortunately, this HD traditional definition is very sensitive to perturbations caused by even a few outlying points, which might result from imperfection in image segmentation. Researchers found that a modified HD is less susceptible to outlying points. ${ }^{9}$ In fact, Equation $C$ replaces Equation B for the directed MHD:

$$
h(M, T)=\frac{1}{p} \sum_{m_{i} \in M} \min _{t_{j} \in T}\left\|m_{i}-t_{j}\right\|,
$$

where $p$ is the number of points in $M$.

However, all HD reported variants do not address each point's unequal contribution. In practice, each point's prominence in representing a facial image will likely be different. In our research, we apply a new HD variant-significance-based multiview Hausdorff distance (SMVHD). This considerably improves the authentication process's robustness even with the introduction of nonrigid distortions to the facial image, such as when a person speaks.

\section{REFERENCES}

1. S. Ben-Yacoub, Y. Abdeljaoued, and E. Mayoraz, "Fusion of Face and Speech Data for Person Identity Verification," IEEE Trans. Neural Networks, vol. 10, no. 5, Sept. 1999, pp. 1065-1074

2. J. Zhang, Y. Yan and M. Lades, "Face recognition: Eigenfaces, Elastic Matching and Neural Nets," Proc. IEEE, vol. 85, no. 9, Sept. 1997, pp. 1422-1435.

3. A. Tefas, C. Kotropoulos, and I. Pitas, "Using Support Vector Machines to Enhance the Performance of Elastic Graph Matching for Frontal Face Authentication," IEEE Trans. PAMI, vol. 23, no. 7, July 2001, pp. 735-746.

4. L. Wiskott et al., "Face Recognition by Elastic Bunch Graph Matching," IEEE Trans. PAMI, vol. 19, no. 7, July 1997, pp. 775-779.

5. W. Zhao et al., Face Recognition: A Literature Survey, tech. report CARTR-948, Univ. Maryland, Center for Automation Research, 2000.

6. M.K.H. Leung and Y.H. Yang, "Dynamic Two-Strip Algorithm in Curve Fitting," Pattern Recognition, vol. 23, nos. 1-2, Jan./Feb., 1990, pp. 69-79.

7. D.P. Huttenlocher, G.A. Klanderman, and W.J. Rucklidge, "Comparing IMAGES USING the Hausdorff Distance," IEEE Trans. PAMI, vol. 15, no. 9, Sept. 1993, pp. 850-863.

8. R.W. Frischolz and U. Dieckmann, "BiolD: a Multimodal Biometric Identification System," Computer, vol. 33, no. 2, Feb. 2000, pp. 64-69.

9. M.P. Dubuisson and A.K. Jain, "A Modified Hausdorff Distance for Object Matching," Proc. 12th Int'I Conf. Pattern Recognition, IEEE CS Press, Los Alamitos, Calif., 1994, pp. 566-568.

$$
\begin{aligned}
& b_{S M V H D}(T, M)=\frac{1}{\sum_{k=1}^{n} \sum_{t_{i}^{k} \in T^{k}} \operatorname{Sig}_{t_{i}^{k} m_{j}^{k}}} \\
& \quad \times \sum_{k=1}^{n} \sum_{t_{i}^{k} \in T^{k}}\left(\operatorname{Sig}_{t_{i}^{k} m_{j}^{k}} \bullet \min _{m_{j}^{k} \in M^{k}}\left\|t_{i}^{k}-m_{j}^{k}\right\|\right),
\end{aligned}
$$

where $\operatorname{Sig}_{m_{i}^{k} t_{j}^{k}}=\frac{1}{2}\left(\operatorname{Sig}_{m_{i}^{k}}+\operatorname{Sig}_{t_{j}^{k}}\right)$ is the average significance (within the range $0 \ldots \infty$ ) of point $m_{i}^{k}$ and its corresponding point $t_{j}^{k}$.
We derive the significance as follows. We apply the dynamic two-strip algorithm ${ }^{12}$ to detect dominant points, which are the points with high curvatures, on the facial edge curves. In the Dyn2S algorithm, we fit a strip to the left and right of each point on the curve and approximate the points inside each strip as a straight line (see Figure 3). The strip's orientation and width adjusts automatically, favoring longer and narrower strips. We compute each curvature's point as the angle subtended by its two strips. A strip's elongatedness is defined as the ratio of its length and width. Then, we calculate a measure of merit based on the strips' lengths and widths, and the angle between the strips. We can compute a point's merit as $E^{\text {left }} . S$. $E^{\text {right }}$, where $S=\left|180^{\circ}-\theta\right|$ is the acuteness of the angle $\theta$ between the two strips, and $E^{\text {left }}$ and $E^{\text {right }}$ are the left and right strips' elongatedness. The algorithm chooses minimum and maximum strip widths. 


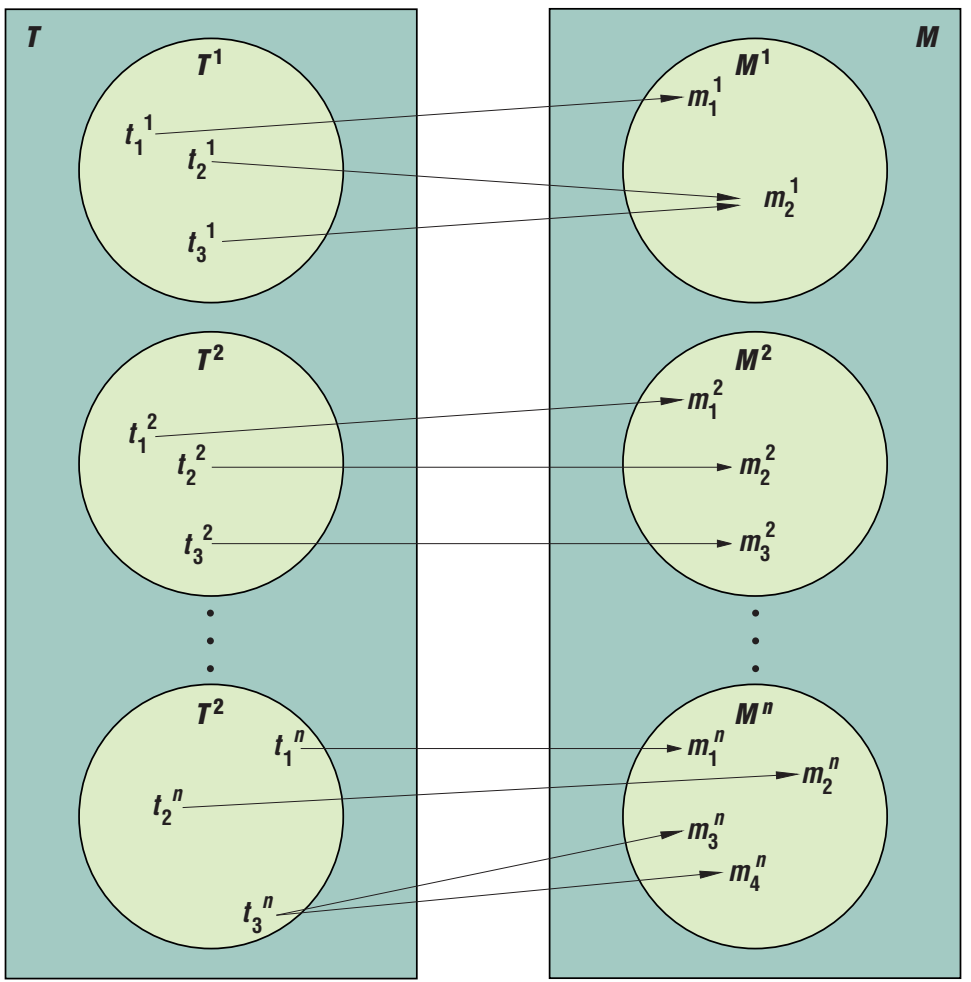

We apply local maximum detection to select points of high curvature. An initial strip of minimal width (the dashed line in Figure 3 ) extends from $P$ to encompass as many points as possible to one side. Its width is incremented and its orientation adjusts to include as many points as possible until it reaches the maximum strip width (the left solid-line strip). The elongatedness measure for each strip is recorded. We apply the same procedure to $P$ 's other side and retain the left and right strips with the highest elongatedness measures. The algorithm chooses a subset of significant points using local maxima. It selects them in three steps. First, it eliminates points with small merit, as compared to their neighbors. Then, it reinstates a number of points to avoid overelimination. It chooses the points from any points not covered by a strip selected in the first step. Finally, it deletes points that align approximately on a straight line, except for the curve's two endpoints. The remaining points are the feature points extracted.

This measure of merit provides an objective evaluation of each point's prominent strength, and we can use it as a significance measure of the dominant point in the proposed distance measure. Thus, the significance measure is derived automatically as a by-product of the feature point detection algorithm.

Compared to other HD variants, where the contributions from all points are equal, every

$$
\min _{t_{j}^{k} \in T^{k}}\left\|m_{i}^{k}-t_{j}^{k}\right\|
$$

(that is, a matched pair's distance) in SMVHD is weighted by the average significance of $m_{i}^{k}$ and $t_{j}^{k}$ because its contribution to $h_{S M V H D}(M, T)$ is assumed to be proportional to the two matched points' significances. The same property applies to $h_{S M V H D}(M, T)$. In fact, SMVHD is symmetric with respect to $M$ and T. So, matching $M$ to $T$ will yield the same result as matching $T$ to $M$. Additionally, we can match multiple points in $M$ to a single point in $T$ and vice versa (see Figure 2). This introduces tolerance to nonrigid distortion into the distance measure.
Figure 2. Significance-based multiview Hausdorff distance matching.

\section{Multiview authentication system}

We developed an experimental access control system based on fusing multiple views of facial images using SMVHD. Figure 1 shows a possible application scenario of the system, where a stationary person stands at a designated position to have multiple facial images taken. We can obtain the multiple views either by installing multiple fixed cameras at the desired locations (three in our case) or by using a single camera that moves on a track. With suitable hardware components, you could use the system for other authentication purposes, such as online business transactions.

During enrollment, as shown in Figure $1 \mathrm{a}$, we capture three views (frontal, threequarter, and profile) as still images. Preprocessing isolates the facial area to reduce the effect of hair and clothing. For the profile image, preprocessing entails nose tip and chin detection, ${ }^{13}$ followed by facial area normalization, alignment, and cropping. For other images, the preprocessing procedure is the same except that we perform eye detection ${ }^{14}$ instead of nose tip and chin detection. We store the extracted model points in a database as $M_{X}{ }^{k}$ for person $X$ with views $k=1,2,3$.

During authentication, anyone who claims to be person $X$ has multiple views captured as still images, which are preprocessed with the same procedure as in the enrollment. This results in $T_{X ?^{k}}$ for the person claiming to be person $X$. Based on SMVHD matching of the stored model for person $X\left(M_{X}{ }^{k}\right)$, the system decides whether this is in fact person $X$. For generality, our system also permits a final decision made by combining results from other analysis modes (for example, speech). However, we haven't implemented this because we are interested in optimizing multiview facial analysis performance, which is essentially a single-modal operation. 
Figure 3. Illustration of the dynamic twostrip algorithm. ${ }^{13}$ An initial strip (the dashed line) of minimal width extends from $P$ to encompass as many points as possible to one side.

\section{Performance analysis}

Here, we analyze our system's performance. Our experiments' results have been very encouraging.

\section{Modified HD and SMVHD matching}

We compared MHD (see the "Face Recognition" sidebar) and SMVHD, using images from different sources: the Stirling_faces, nott-faces-originals, ${ }^{15}$ and images we took. Stirling_faces is a collection of 311 images of 35 persons. It contains 31 complete sets of images $(16 \mathrm{fe}$ males and 15 males), each with three poses (frontal, three-quarter, and profile) and three expressions (neutral, smiling, and speaking). Figure 4 show an example for one person in the database.

Similarly, the nott-faces-originals collection contains 384 images with each face in four expressions (neutral, smiling,

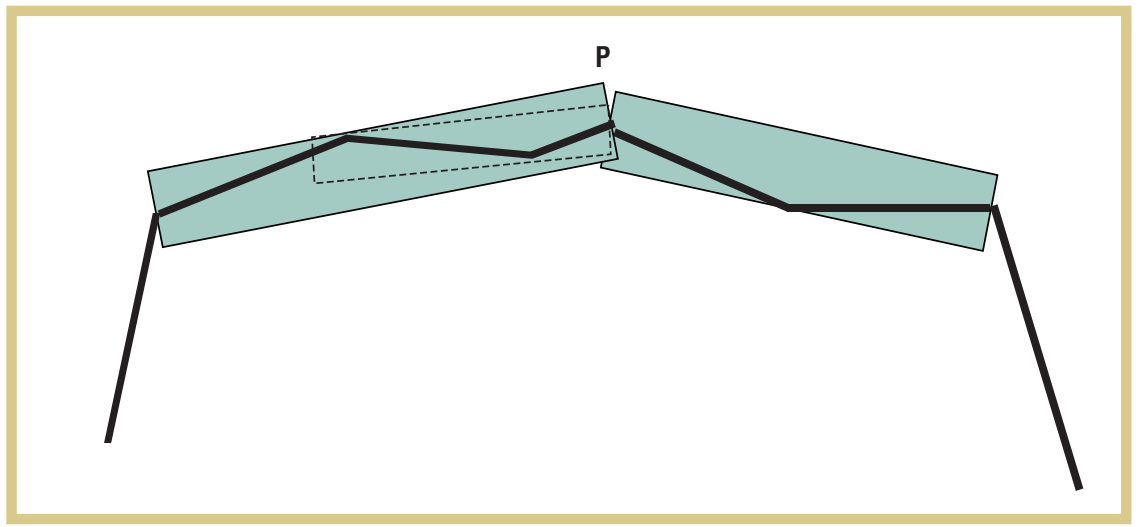

surprise, and disgust) with a frontal view, two expressions (neutral and smiling) with a three-quarter view, and one frontal view wearing a bathing cap over hair. In this case, we selected 88 images of 20 individuals (neutral frontal with or without bathing cap, smiling frontal, neutral three-quarter, and smiling three-quarter). This collection poses two particular challenges. First, the algorithms (especially, SMVHD) must operate on fewer images (for example, without the profile view). Second, the bathing cap obviously causes more difficulty. We augmented these collections with 360 images of 40 individuals of our own.
We compared MHD and SMVHD's matching results using these images. Table 1 summarizes the results. Our experiments show that SMVHD performs significantly better than MHD in terms of accuracy. We achieved a 100 percent match with faces without nonrigid distortions (neutral expressions). Even with nonrigid distortions (smiling and speaking), SMVHD still achieved very high matching accuracy. We also measured the computational times in these experiments. We used an SGI Octane workstation with a 300-MHz CPU and 512 Mbytes RAM. On average, SMVHD required 0.52 s per person (all three views), and MHD required $0.22 \mathrm{~s}, 0.19 \mathrm{~s}$, and $0.12 \mathrm{~s}$ for the

Figure 4. One person's facial images from the Stirling database.

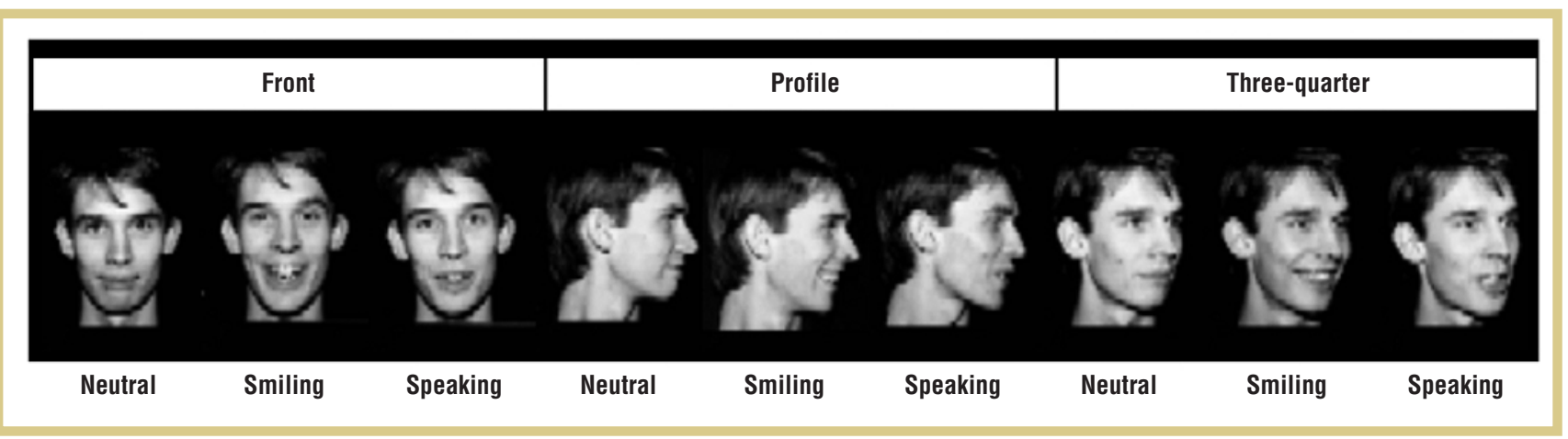

TABLE 1

Comparisons of the accuracy of modified Hausdorff distance and significance-based multiview HD.

\begin{tabular}{|l|c|c|c|c|}
\cline { 2 - 5 } & \multicolumn{2}{|c|}{ MHD } & SMVHD \\
\hline Expression & Frontal view (\%) & Three-quarter View (\%) & Profile view (\%) & All views, fused (\%) \\
\hline Neutral & 96.5 & 96.0 & 90.0 & 100.0 \\
\hline Smiling & 71.1 & 80.5 & 64.5 & 93.6 \\
\hline Speaking & 87.1 & 80.6 & 58.1 & 93.5 \\
\hline
\end{tabular}




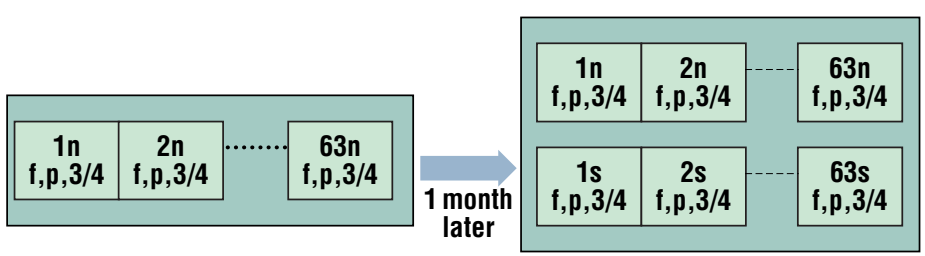

(a) (b)

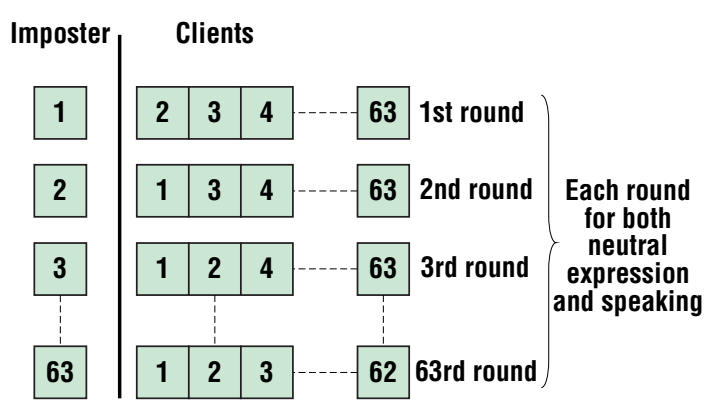

(c)

Figure 5. Experimental protocol: Image capture for (a) training neutral expression, $n$, for enrollment and (b) testing $n$ and speaking, s, for authentication. (Each of the 63 persons had frontal, f; profile, p; and three-quarter, 3/4, views taken.) (c) A leave-one-out and rotation scheme.

frontal, three-quarter, and profile views. So, the average computational time per person was about half a second in each case.

\section{Authentication performance}

In this experiment, we aimed to simulate real-life situations for access control as much as possible. This required a delay between images captured for the enrollment process and authentication. Our experimental protocol follows. Sixty-three volunteers took part-41 males and 22 females. During the enrollment process, each person had three images captured (frontal, three-quarter, and profile), all with neutral expressions. One month later, we captured the images of the same 63 people for authentication. We took three shots of each person (frontal, three-quarter, and profile), first with a neutral expression, followed by nonrigid distortions caused by speaking (saying their name). Introducing nonrigid distortion would allow for mixedmode processing with speech analysis if desired. We also randomly altered the illumination to simulate a typical real-life environment. We conducted our experiments in a laboratory with windows on one side, and we varied the mix of ambient sunlight with fluorescent lights.

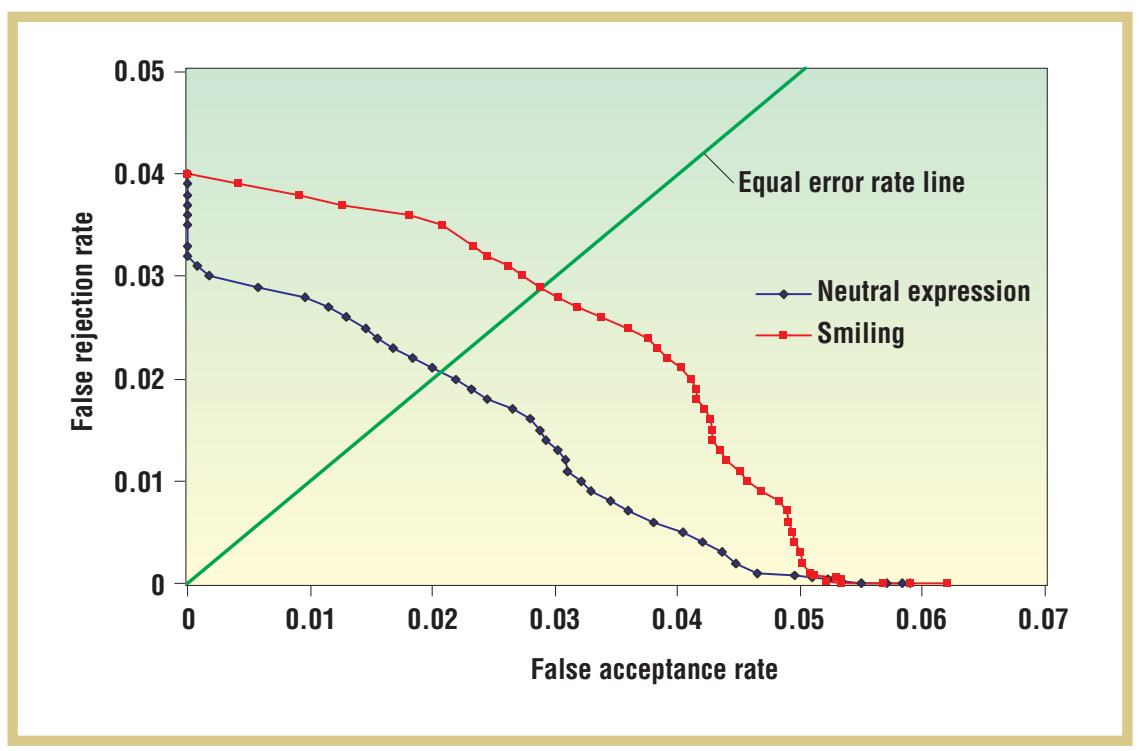

We conducted the experiments using a "leave-one-out and rotation" scheme. For this, we labeled a person an "imposter," with others acting as "clients." The imposter attempted to be identified as one of the 62 clients. The number of times that each imposter gained access under someone else's identity contributed to the FAR. The clients also tried to gain access under their own identity, contributing to the FRR. We obtain the FAR by dividing the number of imposter acceptances by the number of imposter claims; we obtain the FRR by dividing the number of client rejections by the number of client claims. Figure 5 diagrams the experimental protocol. Figure 6 gives a ROC plot for our experimental system.

Figure 6 shows that we can achieve an EER of about 0.022 even with the variation in illumination during authentication. This result far surpasses single-modal operations that rely on facial analysis reported elsewhere. ${ }^{11,16,17}$ In fact, this performance level already approaches those of multimodal methods. ${ }^{11}$ Even with nonrigid distortion caused by speaking during authentication, the EER of about 0.029 was still reasonably good.

Figure 6. Receiver operating characteristic plot for our experimental system. 
F urther research is underway to finetune our algorithm and to conduct more tests on the system. In particular, we would like to measure our system's stability. Through a bootstrap process, we can leave out some views of each person during the enrollment process and subsequently present those unseen views during authentication. We can then repeat this process by leaving out an additional set of views. This should result in both an average score and a variance measure, which would let us better gauge the process's stability, especially when performed daily over a period of time. P

\section{ACKNOWLEDGMENTS}

We thank Roy Want and the anonymous reviewers for their constructive comments and advice, which helped us improve this article's presentation and contents.

\section{REFERENCES}

1. M. Weiser, "The Computer of the 21st Century," Scientific American, vol. 265, no. 3, Sept. 1991, pp. 66-75.

2. R. Want et al., "Disappearing Hardware," IEEE Pervasive Computing, vol. 1, no 1, Jan.-Mar. 2002, pp. 36-47.

3. T. Kindberg and A. Fox, "System Software for Ubiquitous Computing," IEEE Pervasive Computing, vol. 1, no. 1, Jan.-Mar. 2002, pp. 70-81.

4. M. Satyanarayanan, "Challenges for Pervasive Computing: Vision and Challenges," IEEE Personal Communications, vol. 8 no. 4, Aug. 2001, pp. 10-17.

5. A.C.M. Fong and G.Y. Hong, "Implanted Digital Identity," The IPCom Journal, vol. 1, no. 3, Mar. 2001. p. 29.

6. S. Pankanti, R. M. Bolle, and A. Jain, "Biometrics: The Future of Identification," Computer, vol. 33, no. 2, Feb. 2000, pp. 46-49.

7. S. Liu and M. Silverman, "A Practical Guide to Biometric Security Technology," IEEE IT Pro, vol. 3, no. 1, Jan./Feb. 2001, pp. 27-32.
8. P. J. Phillips et al., "An Introduction to Evaluating Biometric Systems," Computer, vol. 33, no. 2, Feb. 2000, pp. 56-63.

9. A. Tefas, C. Kotropoulos, and I. Pitas, "Using Support Vector Machines to Enhance the Performance of Elastic Graph Matching for Frontal Face Authentication," IEEE Trans. PAMI, vol. 23, no. 7, July 2001, pp. 735-746.

10. C. Kotropoulos, A. Tefas, and I. Pitas, "Frontal Face Authentication Using Morphological Elastic Graph Matching," IEEE Trans. Image Proc., vol. 9, no. 4, Apr. 2000, pp. 555-560.

11. S. Ben-Yacoub, Y. Abdeljaoued, and E. Mayoraz, "Fusion of Face and Speech Data for Person Identity Verification," IEEE Trans. Neural Networks, vol. 10, no. 5, Sept. 1999, pp. 1065-1074.

12. M.K.H. Leung and Y.H. Yang, "Dynamic Two-Strip Algorithm in Curve Fitting," Pattern Recognition, vol. 23, nos. 1-2, Jan./ Feb. 1990, pp. 69-79.

13. Y. Gao, Human Face Recognition Using Line Edge Information, doctoral dissertation, Nanyang Technological Univ., Singapore, 2000.

14. K.M. Lam and H. Yan, "Locating and Extracting the Eye in Human Face Images," Pattern Recognition, vol. 29, no. 5, May 1996, pp. 771-779.

15. University of Stirling Face Database, http://pics.psych.stir.ac.uk or http://pics. psych.stir.ac.uk/cgi-bin/PICS/New/pics. cgi? filename $=$

16. J. Zhang, Y. Yan and M. Lades, "Face Recognition: Eigenfaces, Elastic Matching and Neural Nets," Proc. IEEE, vol. 85, no. 9, Sept. 1997, pp. 1422-1435.

17. B. Duc, S. Fischer, and J. Bigun, "Face Authentication with Gabor Information on Deformable Graphs," IEEE Trans. Image Proc., vol. 8, no. 4, Apr. 1999, pp. 504-516.

For more information on this or any other computing topic, please visit our Digital Library at http:// computer.org/publications/dlib.
Useful Reading

R. Chellappa, C.L. Wilson, and S. Sirohey, "Human and Machine Recognition of Faces," Proc. IEEE, vol. 83, no. 5, May 1995, pp. 705-740.

\section{the AUTHORS}

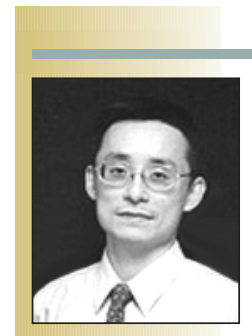

Yongsheng Gao is a lecturer with the School of Microelectronic Engineering at Griffith University. His research interests include face recognition, biometrics, image retrieval, computer vision, and pattern recognition. He received his $\mathrm{BSC}$ and $\mathrm{MSc}$ in electronic engineering from Zhejiang University, China, and $\mathrm{PhD}$ in computer engineering from Nanyang Technological University, Singapore. $\mathrm{He}$ is a member of the IEEE. Contact him at the School of Microelectronic Eng., Room: Technology 3.25, Griffith Univ., Kessels Rd, QLD 4111, Australia; yongsheng.gao@griffith.edu.au.

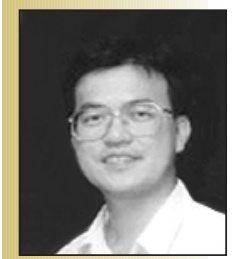

S.C. Hui is an associate professor in the School of Computer Engineering at Nanyang Technological University. His research interests include data mining, Internet technology, and multimedia systems. He received a BSc in mathematics and a DPhil in computer science from the University of Sussex, UK. He is a member of IEEE and ACM. Contact him at the School of Computer Eng., Nanyang Technological Univ., Blk. N4, \#02A-32, Nanyang Ave., Singapore 639798; asschui@ntu.edu.sg.

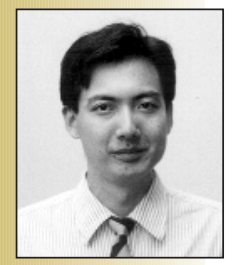

A.C.M. Fong is a lecturer at Massey University. His research interests include various aspects of Internet technology, information theory, and video and image signal processing. He received a BEng in electronic and elec-

trical engineering and computer science and an $\mathrm{MSc}$ in EEE from Imperial College, London, and a PhD in EEE from the University of Auckland. $\mathrm{He}$ is a member of the IEEE and IEE, and is a chartered engineer. Contact him at the Inst. of Information and Mathematical Sciences, Massey Univ., Albany Campus, Private Bag 102-904, North Shore Mail Centre, Auckland, New Zealand; a.c.fong@massey.ac.nz. 